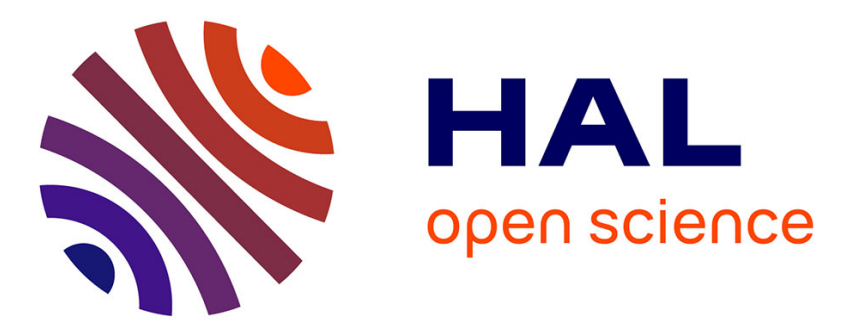

\title{
GMRT discovery of PSR J1544+4937, an eclipsing black-widow pulsar identified with a Fermi LAT source
}

\author{
B. Bhattacharyya, J. Roy, P. S. Ray, Y. Gupta, R. W. Romani, S. M.
}

Ransom, E. C. Ferrara, M. T. Wolff, F. Camilo, Ismaël Cognard, et al.

\section{- To cite this version:}

B. Bhattacharyya, J. Roy, P. S. Ray, Y. Gupta, R. W. Romani, et al.. GMRT discovery of PSR J1544+4937, an eclipsing black-widow pulsar identified with a Fermi LAT source. The Astrophysical journal letters, 2013, 773 (1), 6 pp. 10.1088/2041-8205/773/1/L12 . insu-01261369

\section{HAL Id: insu-01261369 \\ https://hal-insu.archives-ouvertes.fr/insu-01261369}

Submitted on 24 Jun 2016

HAL is a multi-disciplinary open access archive for the deposit and dissemination of scientific research documents, whether they are published or not. The documents may come from teaching and research institutions in France or abroad, or from public or private research centers.
L'archive ouverte pluridisciplinaire HAL, est destinée au dépôt et à la diffusion de documents scientifiques de niveau recherche, publiés ou non, émanant des établissements d'enseignement et de recherche français ou étrangers, des laboratoires publics ou privés. 


\title{
GMRT DISCOVERY OF PSR J1544+4937: AN ECLIPSING BLACK-WIDOW PULSAR IDENTIFIED WITH A FERMI-LAT SOURCE
}

\author{
B. Bhattacharyya ${ }^{1}$, J. Roy ${ }^{1}$, P. S. Ray ${ }^{2}$, Y. Gupta ${ }^{1}$, D. Bhattacharya ${ }^{3}$, R. W. Romani ${ }^{4}$, S. M. Ransom ${ }^{5}$, E. C. Ferrara ${ }^{6}$,

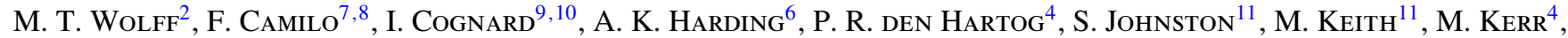 \\ P. F. Michelson ${ }^{4}$, P. M. Saz Parkinson ${ }^{12}$, D. L. Wood ${ }^{13,14}$, and K. S. Wood ${ }^{2}$ \\ ${ }^{1}$ National Centre for Radio Astrophysics, Tata Institute of Fundamental Research, Pune 411 007, India \\ ${ }^{2}$ Space Science Division, Naval Research Laboratory, Washington, DC 20375-5352, USA \\ ${ }^{3}$ Inter-University Centre for Astronomy and Astrophysics, Pune 411 007, India \\ ${ }^{4}$ W. W. Hansen Experimental Physics Laboratory, Kavli Institute for Particle Astrophysics and Cosmology, Department of Physics and \\ SLAC National Accelerator Laboratory, Stanford University, Stanford, CA 94305, USA \\ ${ }^{5}$ National Radio Astronomy Observatory (NRAO), Charlottesville, VA 22903, USA \\ ${ }^{6}$ NASA Goddard Space Flight Center, Greenbelt, MD 20771, USA \\ ${ }^{7}$ Columbia Astrophysics Laboratory, Columbia University, New York, NY 10027, USA \\ ${ }^{8}$ Arecibo Observatory, Arecibo, Puerto Rico, PR 00612, USA \\ ${ }^{9}$ Laboratoire de Physique et Chimie de l'Environnement, LPCE UMR 6115 CNRS, F-45071 Orléans Cedex 02, France \\ ${ }^{10}$ Station de radioastronomie de Nançay, Observatoire de Paris, CNRS/INSU, F-18330 Nançay, France \\ ${ }^{11}$ CSIRO Astronomy and Space Science, Australia Telescope National Facility, Epping, NSW 1710, Australia \\ ${ }^{12}$ Santa Cruz Institute for Particle Physics, Department of Physics and Department of Astronomy and Astrophysics, \\ University of California at Santa Cruz, Santa Cruz, CA 95064, USA \\ ${ }^{13}$ Praxis Inc., Alexandria, VA 22303, USA \\ ${ }^{14}$ Naval Research Laboratory, Washington, DC 20375, USA \\ Received 2013 April 26; accepted 2013 June 24; published 2013 July 29
}

\begin{abstract}
Using the Giant Metrewave Radio Telescope, we performed deep observations to search for radio pulsations in the directions of unidentified Fermi-Large Area Telescope $\gamma$-ray sources. We report the discovery of an eclipsing blackwidow millisecond pulsar, PSR J1544+4937, identified with the uncataloged $\gamma$-ray source FERMI J1544.2+4941. This $2.16 \mathrm{~ms}$ pulsar is in a $2.9 \mathrm{hr}$ compact circular orbit with a very low mass companion $\left(M_{c}>0.017 M_{\odot}\right)$. At $322 \mathrm{MHz}$ this pulsar is found to be eclipsing for $13 \%$ of its orbit, whereas at $607 \mathrm{MHz}$ the pulsar is detected throughout the low-frequency eclipse phase. Variations in the eclipse ingress phase are observed, indicating a clumpy and variable eclipsing medium. Moreover, additional short-duration absorption events are observed around the eclipse boundaries. Using the radio timing ephemeris we were able to detect $\gamma$-ray pulsations from this pulsar, confirming it as the source powering the $\gamma$-ray emission.
\end{abstract}

Key words: binaries: eclipsing - pulsars: general - pulsars: individual (PSR J1544+4937)

Online-only material: color figures

\section{INTRODUCTION}

The Large Area Telescope (LAT; Atwood et al. 2009) on board the Fermi Gamma-ray Space Telescope has discovered a large number of $\gamma$-ray point sources, of which many are unidentified or even unassociated with any known potential counterpart (Ackermann et al. 2012). The LAT can localize most of these sources well enough that they can be covered in a single pointing with the large primary beams of radio telescopes at low frequencies, allowing them to be searched efficiently. Targeted radio searches of unassociated LAT point sources by the Fermi Pulsar Search Consortium (PSC) have resulted in the discovery of 43 radio millisecond pulsars (MSPs; Ray et al. 2012). MSPs are thought to evolve from normal pulsars in binary systems via transfer of angular momentum from companions. Thus, the majority of MSPs are naturally expected to be in binaries $(\sim 83 \%$ being the binary fraction for MSPs in the Galactic field ${ }^{15}$ ). Binary systems where the pulsar wind evaporates the companion are one way to form isolated MSPs. Such systems where the interaction is ongoing are called black-widow (BW) pulsars. Many exhibit long eclipses ( $\sim 10 \%$ of the orbital period, apparently larger than the companion's Roche lobe) that are believed to be caused by the material blown from the very low

\footnotetext{
$\overline{15}$ http://astro.phys.wvu.edu/GalacticMSPs/GalacticMSPs.txt
}

mass companion $\left(M_{c} \ll 0.1 M_{\odot}\right)$ by the pulsar wind. There were two such eclipsing BW systems in the Galactic field known before the launch of Fermi-PSR B1957+20 (Fruchter et al. 1988) and PSR J2051-0827 (Stappers et al. 1996). The BW pulsars are found to have higher values of spin-down energy-loss rate $\left(\dot{E} \sim 10^{34} \mathrm{erg} \mathrm{s}^{-1}\right)$ compared to other MSPs, making these systems good candidates for pulsed $\gamma$-ray emission (Roberts 2011). Among 43 new MSPs found in Fermi-directed searches, there are at least $10 \mathrm{BWs}$ (Ray et al. 2012). This Letter describes the discovery and follow-up study of an eclipsing BW MSP, J1544+4937, with the Giant Metrewave Radio Telescope (GMRT).

\section{OBSERVATIONS AND SEARCH ANALYSIS}

As a part of the PSC search effort, we observed mid- and highGalactic-latitude unassociated Fermi point sources with the GMRT at $607 \mathrm{MHz}$. The GMRT Software Back-end (Roy et al. 2010) produces simultaneous incoherent and coherent filterbank outputs of $512 \times 0.0651 \mathrm{MHz}$ sampled every $61.44 \mu \mathrm{s}$. The wider incoherent beam of the GMRT ( $40^{\prime}$ at $\left.607 \mathrm{MHz}\right)$ can easily cover error circles associated with the Fermi sources. In addition, a coherent beam that is three times more sensitive and narrower (1.5 at $607 \mathrm{MHz}$ using the central core of the GMRT) can be useful if the pulsar happens to be near the pointing center. 
Table 1

Parameters of $\mathrm{J} 1544+4937$

\begin{tabular}{|c|c|}
\hline Parameter & Value $^{\mathrm{a}}$ \\
\hline \multicolumn{2}{|l|}{ Interferometric position $^{b}$} \\
\hline Right ascension (J2000) . . . & $15^{\mathrm{h}} 44^{\mathrm{m}} 04^{\mathrm{s}} .166 \pm 0.3$ \\
\hline Declination $(\mathrm{J} 2000) \ldots$ & $+49^{\circ} 37^{\prime} 57^{\prime \prime} .45 \pm 4^{\prime \prime} .7$ \\
\hline Offset from survey beam center ... & 4.3 \\
\hline \multicolumn{2}{|l|}{ Parameters from radio timing } \\
\hline Right ascension (J2000) ... & $15^{\mathrm{h}} 44^{\mathrm{m}} 04^{\mathrm{s}} .48722(2)$ \\
\hline Declination $(\mathrm{J} 2000) \ldots$ & $+49^{\circ} 37^{\prime} 55^{\prime \prime} .2545(2)$ \\
\hline Position epoch (MJD) ... & 51544.0 \\
\hline Pulsar period, $P(\mathrm{~ms}) \ldots$ & $2.15928839043289(5)$ \\
\hline Pulsar frequency, $f(\mathrm{~Hz}) \ldots$ & $463.11553585462(1)$ \\
\hline Frequency derivative, $\dot{f}\left(\mathrm{~Hz} \mathrm{~s}^{-1}\right) \ldots$ & $-6.29(1) \times 10^{-16}$ \\
\hline Period epoch (MJD) ... & 56007.0 \\
\hline Dispersion measure, $\mathrm{DM}\left(\mathrm{cm}^{-3} \mathrm{pc}\right) \ldots$ & $23.2258(11)$ \\
\hline Binary model ... & ELL1 \\
\hline Orbital period, $P_{b}$ (days) $\ldots$ & $0.1207729895(1)$ \\
\hline Projected semi-major axis, $x$ (lt-s) $\ldots$ & $0.0328680(4)$ \\
\hline Epoch of ascending node passage, $T_{\mathrm{AsC}}(\mathrm{MJD}) \ldots$ & $56124.7701121(2)$ \\
\hline Span of timing data (MJD) ... & 652 \\
\hline Number of TOAs... & 280 \\
\hline Post-fit residual rms $(\mu \mathrm{s}) \ldots$ & 6.9 \\
\hline Reduced chi-square ... & 2.7 \\
\hline
\end{tabular}

Derived parameters

Mass function, $f\left(M_{\odot}\right) \ldots$

Min companion mass, $m_{c}\left(M_{\odot}\right) \ldots$

0.0000026132

0.017

DM distance ${ }^{c}(\mathrm{kpc}) \ldots$

1.2

Flux density at $322 \mathrm{MHz}(\mathrm{mJy})$...

Flux density at $607 \mathrm{MHz}$ (mJy) ...

Spectral index ...

Surface magnetic field, $B_{s}\left(10^{8} \mathrm{G}\right) \ldots$

Spin-down luminosity, $\dot{E}\left(10^{34} \mathrm{erg} \mathrm{s}^{-1}\right) \ldots$

Characteristic age, $\tau$ (Gyr) ...

$\gamma$-ray parameters ${ }^{\mathrm{d}}$

Photon flux $\left(>0.1 \mathrm{GeV}, \mathrm{cm}^{-2} \mathrm{~s}^{-1}\right) \ldots$

Energy flux $\left(>0.1 \mathrm{GeV}\right.$, erg $\left.\mathrm{cm}^{-2} \mathrm{~s}^{-1}\right) \ldots$

Luminosity, $L_{\gamma} / f_{\Omega}\left(>0.1 \mathrm{GeV}\right.$, erg cm $\left.\mathrm{cm}^{-2} \mathrm{~s}^{-1}\right) \ldots$

Efficiency, $\eta_{\gamma} / f_{\Omega}(>0.1 \mathrm{GeV}) \ldots$

5.4

1.2

$-2.3$

0.805 (1)

$1.150(8)$

$11.65(3)$

Notes.

${ }^{a}$ Errors in the last digit are in parentheses.

b Roy et al. (2012).

c Cordes \& Lazio (2002).

${ }^{\mathrm{d}}$ Phase averaged.

One of the targets was FERMI J1544.2+4941, a $\gamma$-ray source from an unpublished internal source list created by the LAT Collaboration using 18 months of data in preparation for the 2FGL catalog (Nolan et al. 2012). The source location (J2000) from that analysis (and used for our telescope pointing) was R.A. $=236.074$, decl. $=49.695$, with a $95 \%$ confidence error circle of radius 9'.5. This source is very weak, with a likelihood test statistic (TS; Mattox et al. 1996) of 26.2 in the 18 month analysis, and did not make the significance cut to be included in the 2FGL catalog itself.

We processed the data on an IUCAA HPC cluster with Fourier-based acceleration search methods using PRESTO (Ransom et al. 2002). We investigated trial dispersion measures (DMs) ranging from $0 \mathrm{pc} \mathrm{cm}^{-3}$ up to $350 \mathrm{pc} \mathrm{cm}^{-3}$. A linear drift of up to 200 Fourier-frequency bins for the highest summed harmonic was allowed. The powerline, $50 \mathrm{~Hz}$, and its subsequent harmonics were excised. Using parameters of $32 \mathrm{MHz}$ band-

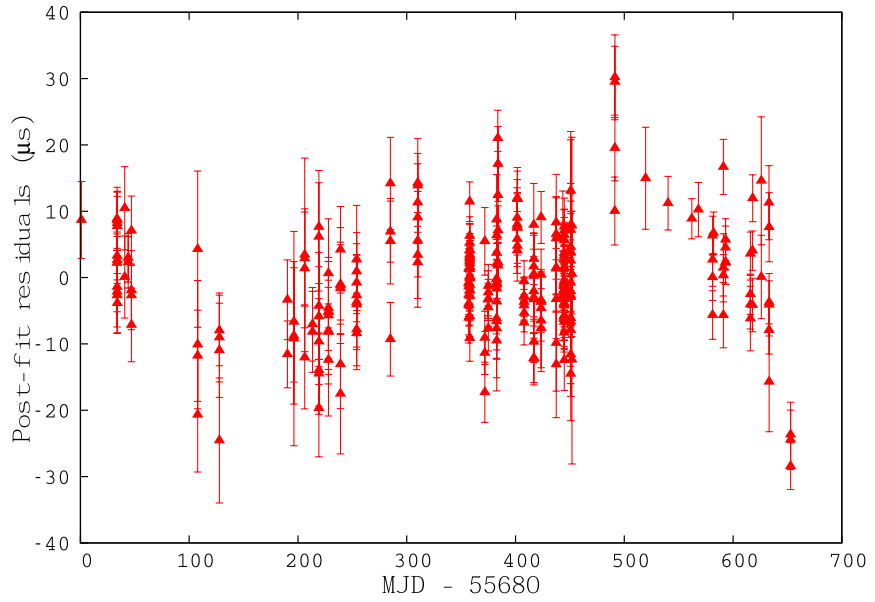

Figure 1. Post-fit timing residuals of J1544+4937 considering non-eclipsing binary phases.

(A color version of this figure is available in the online journal.)

width, $10 \%$ duty-cycle, incoherent array gain of $2.3 \mathrm{~K} \mathrm{Jy}^{-1}$, for 30 minutes of observing, we estimate the search sensitivity as $\left(92 \mathrm{~K}+T_{\text {sky }}\right) /(335 \mathrm{~K}) \mathrm{mJy}$ for a $5 \sigma$ detection at $607 \mathrm{MHz}$. Considering $|b|>5^{\circ}$, where $T_{\text {sky }} \sim 10-45 \mathrm{~K}$, our search sensitivity is $0.3-0.4 \mathrm{mJy}$.

In a 30 minute pointing on 2011 February 1, toward FERMI $\mathrm{J} 1544.2+4941$ we discovered a binary MSP of period $2.16 \mathrm{~ms}$ with significant acceleration of $2.25 \mathrm{~m} \mathrm{~s}^{-2}$ at a DM of $23.2 \mathrm{pc} \mathrm{cm}^{-3}$.

\section{FOLLOW-UP TIMING}

We localized J1544+ 4937 with an accuracy of 5" (positions listed in Table 1) using continuum imaging for the full GMRT array followed by multi-pixel beamforming (Roy et al. 2012), which allowed us to have sensitive follow-up studies using the coherent array. We estimate a flux of $5.4 \mathrm{mJy}$ at $322 \mathrm{MHz}$, and a spectral index of -2.3 . We started the regular timing campaign for $\mathrm{J} 1544+4937$ in 2011 April at $322 \mathrm{MHz}$ with the same coherent filter bank. With the derived position from the multi-pixel search and an a priori binary model predicted by Bhattacharyya \& Nityananda (2008), we obtained phaseconnected time-of-arrivals (TOAs) from TEMPO, ${ }^{16}$ using the JPL DE405 solar system ephemeris (Standish 2004). The binary timing model used is ELL1 (Lange et al. 2001), since $\mathrm{J} 1544+4937$ is in a very low eccentricity system. This MSP is in a very compact binary with an orbital period of $2.9 \mathrm{hr}$. We derive a minimum companion mass (for $90^{\circ}$ orbital inclination) of $0.017 M_{\odot}$ using the Keplerian mass function, assuming a pulsar mass of $1.4 M_{\odot} . \mathrm{J} 1544+4937$ is eclipsed for about $13 \%$ of the orbit at $322 \mathrm{MHz}$ (Section 6). The best-fit timing model (MJD 55680.927-56332.90) is obtained excluding the TOAs around the eclipse phase $(0.05-0.35)$. We achieved a post-fit rms timing residual of $6.9 \mu$ s from 652 days of timing (Figure 1). There are still unmodeled residuals, which can be partially absorbed by proper motion fit. However, the inclusion of proper motion reduces the LAT detection significance, indicating that more timing data are required to improve the model. We estimate a precise DM equal to $23.2258(11) \mathrm{pc}^{-3}$ by a timing fit using 322 and $607 \mathrm{MHz}$ TOAs from non-eclipsing binary phases. Ephemeris, position, and derived parameters are listed in Table 1.

\footnotetext{
16 http://tempo.sourceforge.net
} 


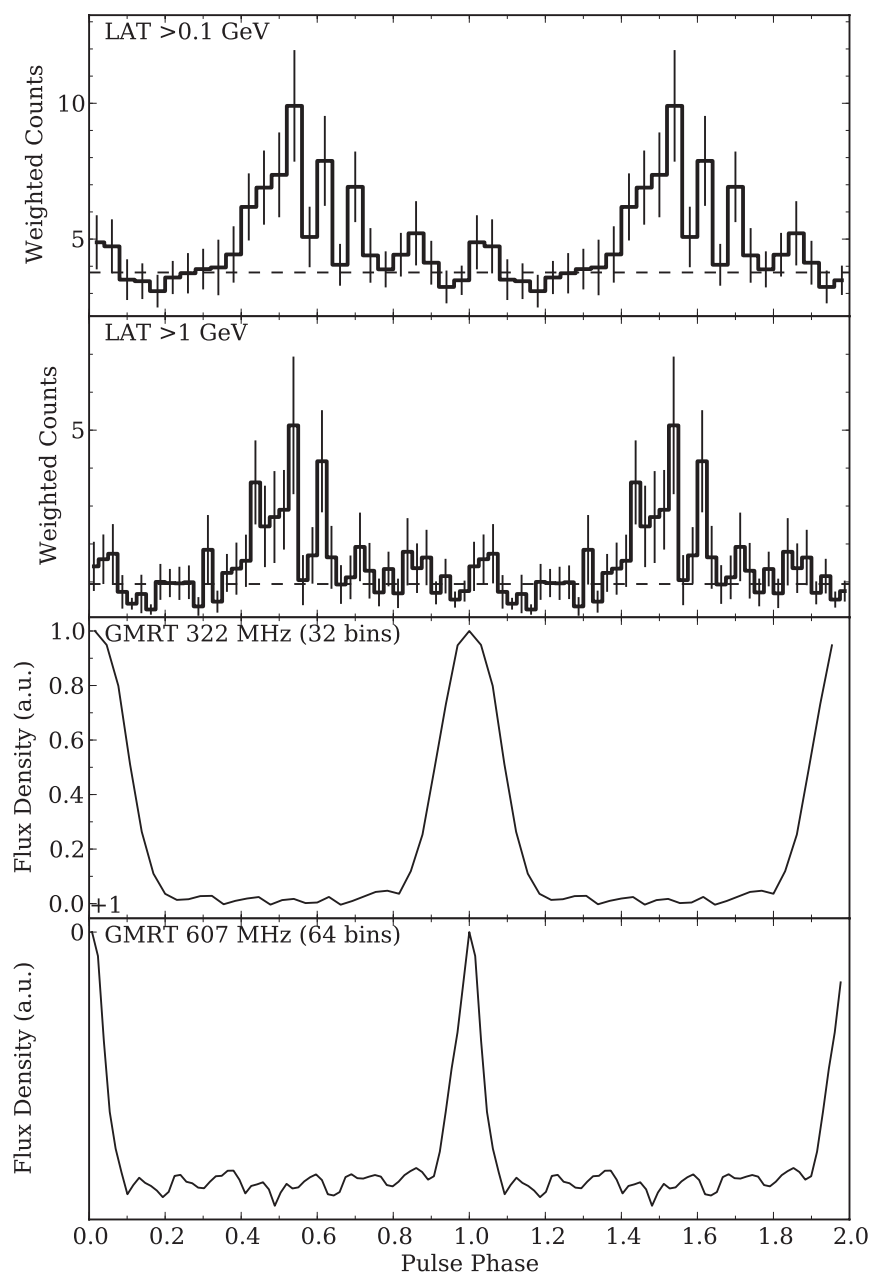

Figure 2. Phase-aligned radio and LAT $\gamma$-ray light curves of J1544+4937. The horizontal dashed lines are an estimate of the background level from sources other than the pulsar. The $322 \mathrm{MHz}$ profile is broadened by incoherent dedispersion across the channel bandwidth of $0.0651 \mathrm{MHz}$, introducing $\sim 373 \mu \mathrm{s}$ smearing.

\section{4. $\gamma$-RAY PULSATIONS}

The radio timing position of $\mathrm{J} 1544+4937$ is 4.3 from the LAT localization of FERMI J1544.2+4941. This is well within the radius of the $95 \%$ confidence error circle, suggesting an association between the pulsar and the LAT source, which we sought to confirm with detection of pulsations. In addition, the $\gamma$-ray detectability metric $\dot{E}^{1 / 2} / d^{2}$ for $\mathrm{J} 1544+4937$ is $7.4 \times 10^{16} \mathrm{erg}^{1 / 2} \mathrm{kpc}^{-2} \mathrm{~s}^{-1 / 2}$, which is comparable to other $\gamma$-ray detected MSPs (Figure 12 of Abdo et al. 2010).

Because of the low significance of the LAT source, a phaseaveraged spectral analysis of the region is required to optimize the sensitivity of the $H$-test for pulsed significance using photon probability weighting (Kerr 2011). We performed a binned likelihood spectral analysis of the region using the Fermi Science Tools. ${ }^{17}$ We selected LAT data with reconstructed energies in the range $100 \mathrm{MeV}$ to $20 \mathrm{GeV}$ collected between 2008 August 4 and 2013 February 6. We used the P7SOURCE_V6 instrument response functions and excluded events at a zenith angle $>100^{\circ}$ and times when the LAT was in the SAA or the rocking angle was greater than $52^{\circ}$. We modeled ${ }^{18}$ a region of radius $15^{\circ}$ using a source list from the $2 \mathrm{FGL}$ catalog with

\footnotetext{
17 http://fermi.gsfc.nasa.gov/ssc/data/analysis/software/

$18 \mathrm{http} / / /$ fermi.gsfc.nasa.gov/ssc/data/access/lat/BackgroundModels.html
}

an additional source added to the location of the pulsar. We modeled the pulsar with an exponentially cutoff power-law spectrum. Because it is so faint, we fitted only for the powerlaw index and normalization, keeping the cutoff energy fixed at $2.5 \mathrm{GeV}$, a value typical of other MSPs (Abdo et al. 2010). The spectral model included both the isotropic and Galactic diffuse contributions with normalizations free in the fit. We detected the source with a TS of 38.2 , corresponding to $\sim 6 \sigma$. The spectral index and cutoff energy are very poorly constrained for this weak source, so to estimate the uncertainty on the integrated flux we repeated the spectral fit with the cutoff frozen at $0.7,2.5$, and $5.0 \mathrm{GeV}$, and with the index frozen at 1.3 (the average value for LAT-detected MSPs) and the cutoff free. We find that the uncertainty due to the spectral shape is about $3 \times 10^{-10} \mathrm{~cm}^{-2} \mathrm{~s}^{-1}$ for the photon flux and $2 \times 10^{-13} \mathrm{erg} \mathrm{cm}^{-2} \mathrm{~s}^{-1}$ for the energy flux. These are smaller than the statistical errors for this faint source and are added in quadrature to compute the errors on the fluxes reported in Table 1. Using the fitted energy flux $(G)$ and the DM distance $(d)$, we compute the $\gamma$-ray luminosity $L_{\gamma}=4 \pi f_{\Omega} G d^{2}$ and efficiency $\eta=L_{\gamma} / \dot{E}$, where the beaming factor $f_{\Omega}$ is assumed to be 1 (Waters et al. 2009).

Using this model for the continuum emission from the region, we search for pulsed $\gamma$-ray emission by selecting events within $2^{\circ}$ of the source and computed the probability that they originated from the source with the Fermi Science Tool gtsrcprob. Using these probabilities as weights and pulse phases computed using the radio timing model, we computed a weighted $H$-test of 37.1, corresponding to a detection significance of $5.1 \sigma$ and confirming that $\mathrm{J} 1544+4937$ is indeed a $\gamma$-ray pulsar and identifying the LAT source as this MSP. The LAT weighted light curves in two energy bands, phase aligned to the radio profiles, are shown in Figure 2. For this faint source, it is difficult to determine the peak multiplicity, but the $\gamma$-ray emission seems to be mainly between phases 0.4 and 0.6 , relative to the single radio peak. Fitting a single Gaussian peak to the light curve gives a peak at phase $0.54 \pm 0.02$ with a width of $0.25 \pm 0.05$.

\section{X-RAY AND OPTICAL OBSERVATIONS}

We analyzed five Swift/X-ray Telescope (XRT) observations (totaling $9.3 \mathrm{ks}$ ) of this field from 2013 March 19-24. No $\mathrm{X}$-ray counterpart is detected, providing a flux upper limit of $\sim 1.7 \times 10^{-14} \mathrm{erg} \mathrm{cm}^{-2} \mathrm{~s}^{-1}$ for $0.3-10 \mathrm{keV}$.

We have checked for an optical counterpart for $\mathrm{J} 1544+4937$ using archival SDSS data. This field was imaged (AdelmanMcCarthy et al. 2008) on MJD 52046.294, at binary phase 0.57. No optical counterpart was detected. The strongest upper limits were $g^{\prime}>22.5, r^{\prime}>22.0$, and $i^{\prime}>21.5$ (90\% CL). We also obtained a $300 \mathrm{~s} \mathrm{H} \alpha$ image with the MiniMo camera on the $3.5 \mathrm{~m}$ WIYN telescope on MJD 55975.521 (S. Brownsberger \& R. W. Romani 2013, in preparation). No stellar counterpart or extended emission was seen with an $\mathrm{H} \alpha$ flux upper limit of $2.5 \times 10^{-16} \mathrm{erg} \mathrm{cm}^{-2} \mathrm{~s}^{-1}(90 \% \mathrm{CL})$ for a compact $<5^{\prime \prime}$ nebula.

Breton et al. (2013) have obtained optical photometry of the heated companions of a number of Fermi MSPs. They find a typical heating efficiency $\eta \sim 0.15$. For systems with a very strong wind $\eta$ approaches unity (e.g., J1810+1744, Breton et al. 2013; J1311-3430, Romani et al. 2012). Breton et al. (2013) give an estimate for the irradiation temperature $T_{\mathrm{Irr}}=$ $\left(\eta \dot{E}_{\mathrm{SD}} / 4 \pi \sigma a^{2}\right)^{1 / 4}$, which is $4370 K$ considering $\eta=0.15$ and $6360 K$ considering $\eta=0.6$ for $\mathrm{J} 1544+4937$. If we assume that the deep, variable radio eclipses imply a near Roche-lobe filling companion, and consider a typical inclination angle $=60^{\circ}$, an unheated (backside) companion temperature of $2500 \mathrm{~K}$ and 

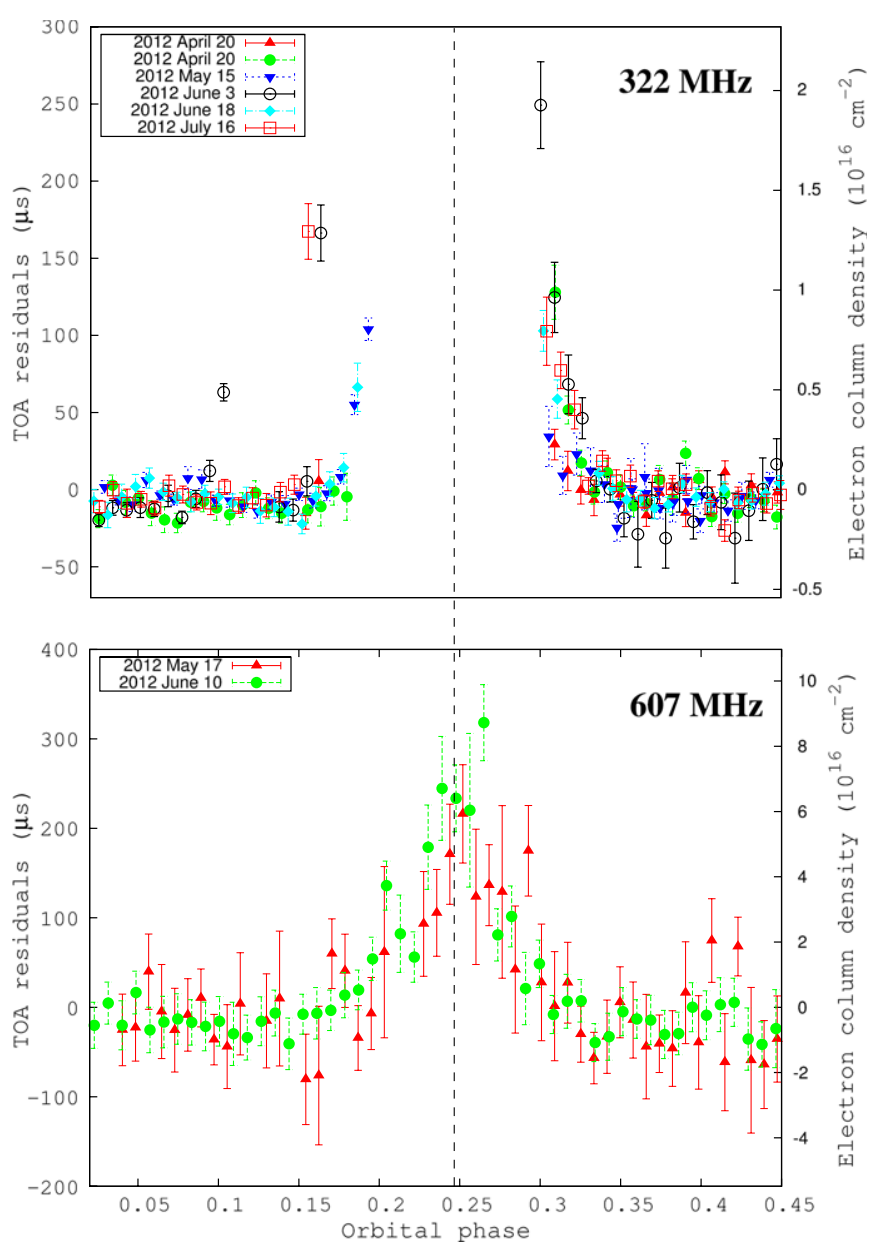

Figure 3. Variation of timing residuals and electron column density (TOAs of $90 \mathrm{~s}$ time resolution) around the eclipse phase at $322 \mathrm{MHz}$ (top) and $607 \mathrm{MHz}$ (bottom).

(A color version of this figure is available in the online journal.)

$\eta=0.15$, we can use the binary light-curve synthesis program "Icarus" (Breton et al. 2012) to predict magnitudes at phase $\phi_{B}=0.57$ of $g^{\prime} \approx 25.2, r^{\prime} \approx 23.9$, and $i^{\prime} \approx 23.1$ (magnitudes at maximum are 23.0, 21.9, and 21.5, respectively). Thus, the observed SDSS limits are not constraining. However, if the irradiation efficiency is higher, the fluxes can be detectable; for example, with $\eta=0.6$ we expect $g^{\prime} \approx 22.4, r^{\prime} \approx 21.8$, and $i^{\prime} \approx 21.6$ at $\phi_{B}=0.57$. These are comparable to our observed magnitude limits, so higher efficiencies are ruled out unless the Roche-lobe filling factor is small or the source distance is larger. These estimates also depend weakly on the observer inclination and the secondary unheated temperature and composition.

Our $\mathrm{H} \alpha$ limit for $\mathrm{J} 1544+4937$ corresponds to $<0.15$ of the highest surface brightness $\sim 5^{\prime \prime}$ patch of the B1957+20 bow shock. The average flux ratio is even more constraining, with an upper limit of $\sim 0.03$ of the total bow-shock flux. However, given the small fraction of MSPs that show optical bow shocks (likely due to the small filling factor of the neutral interstellar medium), the non-detection at such a large distance from the Galactic plane $\left(b \sim 50^{\circ}\right)$ is not surprising.

\section{ECLIPSE CHARACTERISTICS}

Figure 3 presents the timing residuals (and electron column densities) around the eclipse phase at 322 and $607 \mathrm{MHz}$, with
$90 \mathrm{~s}$ time resolution. The effect of the eclipses is generally seen from 0.18 to 0.31 orbital phase (eclipse zone hereafter) at $322 \mathrm{MHz}$. The eclipses are centered at binary phase 0.24 with a duration of around 22 minutes. We estimate the radius of the companion's Roche lobe, $R_{L}$ (Eggleton 1983),

$$
R_{L}=\frac{0.49 a q^{2 / 3}}{0.6 q^{2 / 3}+\ln \left(1+q^{1 / 3}\right)} \sim 0.13 R_{\odot}
$$

where $q=m_{c} / m_{p}$ is the mass ratio of the companion and the pulsar, and $a$ is the separation of the companion from the pulsar ( $a \sim 1.2 R_{\odot}$ for $\mathrm{J} 1544+4937$, indicating an extremely compact binary). The opaque portion of the companion's orbit is $0.98 R_{\odot}$, much larger than $R_{L}$ of $0.13 R_{\odot}$, so the volume occupied by the eclipsing material is well outside the companion's Roche lobe, and thus is not gravitationally bound to the companion. This confirms that this binary is a BW, where the pulsar is ablating its companion, creating a significant amount of intrabinary material that obscures the pulsar's emission.

Our sample consisted of six eclipses at $322 \mathrm{MHz}$ where the pulsar emission was fully obscured by companion and its wind and two $607 \mathrm{MHz}$ observing sessions covering the full orbit (Figure 3). At $607 \mathrm{MHz}$ we detect the MSP throughout the $322 \mathrm{MHz}$ eclipse zone. However, we observe a flux fading at $607 \mathrm{MHz}$ near the superior conjunction (orbital phase 0.24). Significant delays in pulse arrival times are observed at $607 \mathrm{MHz}$ during the eclipse zone at $322 \mathrm{MHz}$. Maximum delay in pulse arrival time at $607 \mathrm{MHz}$ near the eclipse superior conjunction is around $300 \mu \mathrm{s}$, which corresponds to an increase in DM of $0.027 \mathrm{pc} \mathrm{cm}^{-3}$ and an added electron density, $N_{e}$ of $8 \times$ $10^{16} \mathrm{~cm}^{-2}$.

Thompson et al. (1994, T94 hereafter) elucidate a collection of eclipse mechanisms. According to them, eclipsing due to refraction of the radio beam demands an order of magnitude higher group delay ( $\sim$ few tens of milliseconds) than we observe for $\mathrm{J} 1544+4937\left(250 \mu \mathrm{s}\right.$ at $322 \mathrm{MHz}$ egress). Using $N_{e} \sim$ $8 \times 10^{16} \mathrm{~cm}^{-2}$ observed during superior conjunction at $607 \mathrm{MHz}$ and absorption length about twice the size of the eclipse zone, according to Equation (11) of T94, we find that free-free absorption is possible (absorption optical depth $\tau_{\mathrm{ff}}>1$ ) if the plasma temperature $T \leqslant 4 \times f_{\mathrm{cl}}^{2 / 3} \mathrm{~K}$, where $f_{\mathrm{cl}}^{2 / 3}$ is the clumping factor. This demands either a very low temperature or a very high value of the clumping factor, both of which are not physically achievable. Eclipsing by pulse smearing (due to the increase of $N_{e}$ along the line of sight) can be ruled out, as the excess electron column density inferred from $607 \mathrm{MHz}$ predicts $373 \mu$ s smearing of pulses at $322 \mathrm{MHz}$ near superior conjunction (considering incoherent dedispersion), which is less than one-fifth of pulse period. Since J1544+4937 has a narrow main pulse, and no significant profile evolution is apparent at the eclipse boundary, pulse broadening due to scattering can reduce the detectability but cannot explain the eclipse. In addition, since $\mathrm{J} 1544+4937$ is relatively weak, nearby, and has a shallower spectrum than B1957+20, the expected induced Compton scattering optical depth is much less than one (Equation (26) of T94). Another eclipse mechanism considered by T94 is cyclotron-synchrotron absorption of the radio waves by non-relativistic/relativistic electrons, which requires a magnetic field in the vicinity of the companion. We calculate a magnetic field $B \sim 11 \mathrm{G}$ and corresponding cyclotron absorption frequency $\sim 31 \mathrm{MHz}$ (Equations (35) and (37) of T94, assuming a moment of inertia of $10^{45} \mathrm{~g} \mathrm{~cm}^{2}$ ). Thus, 322 and $607 \mathrm{MHz}$ will correspond to 10th and 20th harmonics 


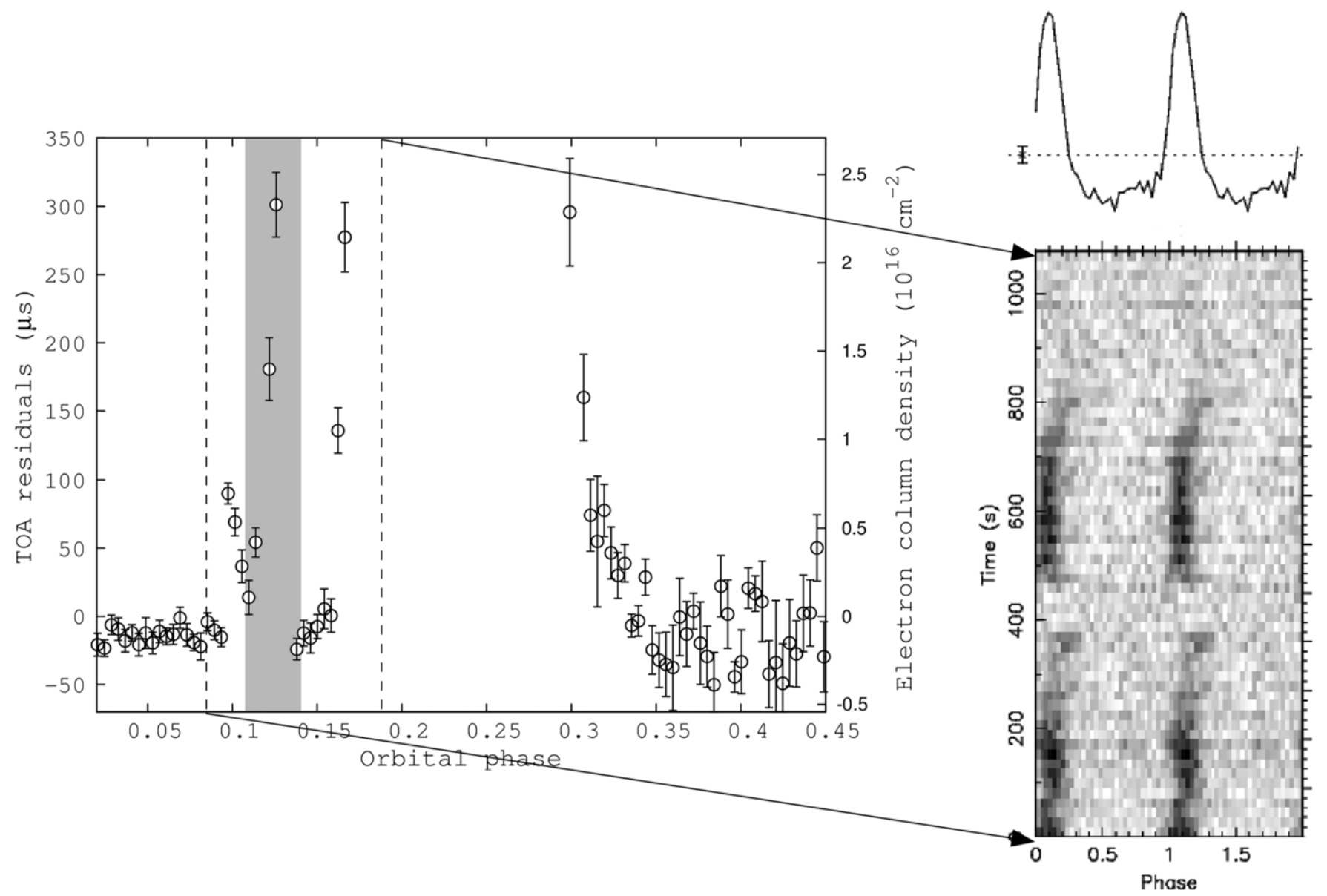

Figure 4. Example of additional short-duration absorption beyond the eclipse phase at $322 \mathrm{MHz}$ on $2012 \mathrm{June}$ 3. Left: variation of timing residuals and electron column density at $42 \mathrm{~s}$ time resolution. Right: phaseogram showing short-duration absorption.

of the cyclotron resonance. For a fixed temperature, the optical depth for cyclotron absorption drops with harmonics, which may explain the lack of absorption seen at $607 \mathrm{MHz}$. A modified model is proposed by Khechinashvili et al. (2000) based on kinematic treatment of cyclotron damping, assuming whitedwarf companions with reasonably strong surface magnetic fields. Further observations over a wider radio spectrum may help to investigate the frequency-dependent degree of damping predicted by this model.

We observe a temporal variation of the ingress phase at $322 \mathrm{MHz}$. For two eclipses, the ingress phase is shifted to 0.16 (from 0.18 for the other four eclipses), whereas there are no apparent shifts in the egress phase. This corresponds to an increase of the opaque portion by $0.15 R_{\odot}$ and $N_{e}>$ $1.2 \times 10^{16} \mathrm{~cm}^{-2}$ at the ingress boundary. Such asymmetric increase of eclipse duration may indicate that our line of sight is probing a wind zone where there is systematic outflow of eclipse material.

We also observe strong phase modulations and additional short-duration absorptions at ingress and egress, in time-series data at higher resolution. The durations of these features are in general around 10-20 s, and hence they are not seen in Figure 3. However, in one of the observing epochs these modulations lasted longer - phase modulation of duration $100 \mathrm{~s}$, followed by a short-duration absorption of $\sim 180 \mathrm{~s}$, then regular emission resumes for $500 \mathrm{~s}$, after which the eclipse starts (Figure 4). Fragmented blobs of plasma randomly oriented around an eclipsing zone, obscuring radiation from the pulsar, can explain these short-duration absorptions.

\section{DISCUSSION}

We report the GMRT discovery of an eclipsing BW pulsar, at the position of an unassociated LAT source, FERMI $\mathrm{J} 1544.2+4941$. This is the first Galactic field MSP discovered at the GMRT. The detection of pulsed $\gamma$-rays from this pulsar demonstrates it as the source powering FERMI J1544.2+4941. Due to the limited significance of the source in $\gamma$-rays, additional data are needed before conclusions on peak multiplicity and system geometry can be drawn. The implied efficiency $(\sim 3 \%)$ of converting spin-down energy into $\gamma$-rays is typical of LAT-detected MSPs. This is the first discovery of a radio MSP in a LAT source fainter than the 2FGL catalog limit (Ray et al. 2012). Since the radio pulsar is relatively bright, this provides strong justification to continue these searches as new LAT sources are revealed in analyses of longer data sets. The radio flux is uncorrelated with the $\gamma$-ray flux (Ackermann et al. 2012), so even faint new LAT sources can harbor bright radio MSPs.

Eclipsing BW pulsars have the potential of providing information on the evolutionary connection between the lowmass X-ray binaries and isolated MSPs. With long-term monitoring of this pulsar, we aim to estimate $\dot{P}_{b}$ and its higher derivatives, which can provide an estimate of the lifespan of the system. Bates et al. (2011) noted that for BW systems the measured value of $\dot{E} / \mathrm{a}^{2}$ is an order of magnitude higher 
than for other MSP binaries, indicative of greater energy flux needed to ablate the companion. For J1544 + 4937 we calculate $\dot{E} / a^{2} \sim 1.5 \times 10^{33} \mathrm{erg}$ lt-s ${ }^{-2} \mathrm{~s}^{-1}$, which is similar to other BW systems in the Galactic field. Dual frequency observations presented in this paper suggest that cyclotron absorption by the plasma formed via interaction of the pulsar wind with ablated material can obscure the pulsed emission. However, exploring the radio spectrum on either side to probe the reduced/increased opaqueness of the stellar wind during the eclipse phase may provide better insight into the plausible eclipse mechanism.

The Fermi-LAT Collaboration acknowledges support from a number of agencies and institutes for both development and the operation of the LAT as well as scientific data analysis. These include NASA and DOE in the United States, CEA/ Irfu and IN2P3/CNRS in France, ASI and INFN in Italy, MEXT, KEK, and JAXA in Japan, and the K. A. Wallenberg Foundation, the Swedish Research Council, and the National Space Board in Sweden. Additional support from INAF in Italy and CNES in France for science analysis during the operations phase is also gratefully acknowledged. We acknowledge the support of the telescope operators of the GMRT, which is run by the National Centre for Radio Astrophysics of the Tata Institute of Fundamental Research. We thank the Swift team at the Pennsylvania State University, especially Abe Falcone. We acknowledge help of C. Cheung in interpreting the XRT data. We thank D. Thompson and T. Johnson for their comments and $\mathrm{R}$. Breton for a discussion of heating fluxes.

\section{REFERENCES}

Abdo, A. A., Ackermann, M., Ajello, M., et al. 2010, ApJS, 187, 460 Ackermann, M., Ajello, M., Allafort, A., et al. 2012, ApJ, 753, 83

Adelman-McCarthy, J., Agüeros, M. A., Allam, S. S., et al. 2008, ApJS, 175,297

Atwood, W. B., Abdo, A. A., Ackermann, M., et al. 2009, ApJ, 697, 1071

Bates, S. D., Bailes, M., Bhat, N. D. R., et al. 2011, MNRAS, 416, 2455

Bhattacharyya, B., \& Nityananda, R. 2008, MNRAS, 387, 273

Breton, R. P. 2013, ApJL, 769, L108

Breton, R. P., Rappaport, S. A., van Kerkwijk, M., et al. 2012, ApJ, 748, 115

Cordes, J. M., \& Lazio, T. J. W. 2002, arXiv:astro-ph/0207156

Eggleton, P. P. 1983, ApJ, 268, 368

Fruchter, A. S., Stinebring, D. R., \& Taylor, J. H. 1988, Natur, 333, 237

Kerr, M. 2011, ApJ, 732, 38

Khechinashvili, D. G., Melikidze, G. I., \& Gil, J. I. 2000, ApJ, 541, 335

Lange, C., Camilo, F., Wex, N., et al. 2001, MNRAS, 326, 274

Mattox, J. R., Bertsch, D. L., Chiang, J., et al. 1996, ApJ, 461, 396

Nolan, P. L., Abdo, A. A., Ackermann, M., et al. 2012, ApJS, 199, 31

Ransom, S. M., Eikenberry, S. S., \& Middleditch, J. 2002, AJ, 124, 1788

Ray, P. S., Abdo, A. A., Parent, D., et al. 2012, in 2011 Fermi Symposium Proceedings, eConf C110509, ed. A. Morselli (arXiv:1205.3089)

Roberts, M. S. E. 2011, in AIP Conf. Proc. 1357, Radio Pulsars: An Astrophysical Key to Unlock the Secrets of the Universe, ed. M. Burgay, N. D'Amico, P. Esposito, A. Pellizzoni, \& A. Possenti (Melville, NY: AIP), 127

Romani, J. 2012, ApJL, 760, L36

Roy, J., Bhattacharyya, B., \& Gupta, Y. 2012, MNRAS, 27, L90

Roy, J., Gupta, Y., Pen, U.-L., et al. 2010, ExA, 28, 55

Stappers, B. W., Bailes, M., Lyne, A. G., et al. 1996, ApJL, 465, L119

Standish, E. M. 2004, A\&A, 417, 1165

Thompson, C., Blandford, R. D., Evans, C. R., \& Phinney, E. S. 1994, ApJ, 422, 304

Watters, K. P., Romani, R. W., Weltevrede, P., \& Johnston, S. 2009, ApJ, 695,1289 